\title{
La nef de l'ancienne cathédrale de Tréguier et la formation d'une architecture gothique régionale
}

Anne-Claude Le Boulc'h

\section{(2) OpenEdition}

\section{Journals}

Édition électronique

URL : http://journals.openedition.org/abpo/1604

DOI : $10.4000 / a b p o .1604$

ISBN : 978-2-7535-1486-7

ISSN : 2108-6443

Éditeur

Presses universitaires de Rennes

Édition imprimée

Date de publication : 20 juin 2002

Pagination : 35-58

ISBN : 978-2-86847-742-2

ISSN : 0399-0826

\section{Référence électronique}

Anne-Claude Le Boulc'h, « La nef de l'ancienne cathédrale de Tréguier et la formation d'une

architecture gothique régionale », Annales de Bretagne et des Pays de l'Ouest [En ligne], 109-2 | 2002,

mis en ligne le 20 juin 2004, consulté le 19 avril 2019. URL : http://journals.openedition.org/abpo/1604 ; DOI : 10.4000/abpo.1604 


\title{
La nef de l'ancienne cathédrale de Tréguier et la formation d'une architecture gothique régionale
}

\author{
Anne-Claude LE BoulC'H \\ chercheur associé, CNRS \\ Laboratoire de médiévistique occidentale de Paris (LAMOP)
}

Entre le XIII ${ }^{\mathrm{e}}$ et le début du XVI ${ }^{\mathrm{e}}$ siècle, une paix relative et une certaine prospérité ont permis la reconstruction d'édifices religieux bretons devenus vétustes ou de dimensions insuffisantes, ainsi que la fondation de nouveaux centres religieux. Cet élan bâtisseur, seulement ralenti par la guerre de Succession, entre 1341 et 1364, s'est accompagné de la formation d'un style propre à la Bretagne encore trop mal connu et qu'il reste à définir maintenant. C'est à partir de la nef de l'ancienne cathédrale de Tréguier, probablement réalisée au cours du XIV ${ }^{\mathrm{e}}$ siècle, que je me propose d'analyser les grands traits de cette architecture, et d'en cerner tant les caractéristiques que l'originalité. La nef de Tréguier, dont les traits se retrouvent dans un grand nombre d'églises bretonnes, semble en effet représentative d'une architecture régionale ${ }^{1}$.

Avant d'aborder ce sujet, il convient d'évoquer quelques problèmes liés à la datation de la nef de Tréguier, problèmes qui se posent du reste pour la plupart des œuvres réalisées en Bretagne au cours du XIV siècle. Puis il conviendra de tenter de reconstituer la chronologie des travaux, d'après les indices architectoniques. Il sera ensuite possible de définir le parti archi-

1. Il me paraît intéressant de citer quelques articles ayant pour sujet l'étude de la cathédrale de Tréguier et de son diocèse, et tout particulièrement celui de Raymond Connon, "La cathédrale de Tréguier ", Congrès Archéologique de France, $107^{\mathrm{e}}$ session (Saint-Brieuc), 1949, p. 102-123, mais aussi CoufFon, René, "Un catalogue des évêques de Tréguier rédigé au Xve siècle ", Mémoires de la Société d'Émulation des Côtes-du-Nord, t. LXI, 1929, p. 33-147. Voir aussi BARRIE, Roger, "La cathédrale Saint-Yves de Tréguier et l'architecture bretonne au XIV ${ }^{\mathrm{e}}$ siècle ", Mémoires de la Société d'Histoire et d'Archéologie de Bretagne, t. LX, 1983, p. 226-294; BARTHELEMY, Anatole de, "Extraits des registres de la fabrique de la cathédrale de Tréguier ", Bulletin du Comité de la Langue, de l'Histoire et des Arts en France, 1852-1853, p. 131 et suivantes, et du même auteur, "Les reliques de saint Tugdual, évêque de Tréguier ", Revue de Bretagne, t. XXV, 1901/1, p. 401-413; MinoIS, Georges, "Culte des saints et vie religieuse dans le diocèse de Tréguier au Xve siècle ", Annales de Bretagne et des Pays de l'ouest, LXXXVII/1, 1980, p. 17-42. 
tectural de cette nef, avant de montrer à quel point celui-ci n'est pas unique, mais se trouve représenté dans la plupart des grands édifices gothiques bretons à la fin du Moyen Âge.

\section{Chronologie relative de la construction de la nef}

La nef de Tréguier fut réalisée au cours du XIve siècle, semble-t-il, mais il reste difficile de situer précisément l'œuvre dans le temps. Nous savons qu'en 1291 le pape accorda une année et quarante jours d'indulgences à tous ceux qui visiteraient l'église à l'une des quatre fêtes de la Vierge ou à la Saint-Tugdual ${ }^{2}$. Cette tentative de réactiver le pèlerinage de Tréguier n'avait-il pas pour but de récolter les fonds nécessaires au lancement d'une nouvelle campagne de construction? D'autres faits sont susceptibles de nous intéresser. Le catalogue des évêques de Tréguier, rédigé au XV siècle $^{3}$, précise que l'évêque Richard du Perrier fit ouvrir un nouveau chantier pour l'édification de la cathédrale, ou d'une partie de la cathédrale ("novum opus ecclesiae "), en 1339. D’après les mêmes sources, en décembre 1342, le pape accorda une indulgence aux pèlerins venant prier sur le tombeau de saint Yves ${ }^{4}$. Vers 1345-1346, les Anglais " rompirent aucunes églises de la Cité afin que leurs adversaires ne s'y fortifiassent, et mesmement partie de l'église cathédrale où repousait Monsieur Saint Yves ${ }^{5}$... ". Enfin, en 1349, eut lieu la translation solennelle du corps de saint Yves dans l'église ${ }^{6}$. Si l'on ajoute à ces renseignements l'hypothèse vraisemblable d'une interruption des travaux au cours de la guerre de Succession, nous possédons une série d'éléments qui nous permettraient peut-être d'avancer quelques suggestions quant à la datation de la nef. Mais ne serait-il pas dangereux d'appuyer une datation de l'œuvre sur une interprétation peutêtre trop hasardeuse de ces renseignements? Il est vraisemblable que seule une analyse minutieuse de l'architecture, et un travail de comparaison systématique, permettraient d'avancer sur un terrain plus solide.

La nef gothique de Tréguier fut édifiée à l'ouest d'un chœur et d'un transept romans, dont il subsiste un témoin, la tour Hasting ${ }^{7}$. Celle-ci, probablement située à l'extrémité du bras nord du transept roman, ne fut pas démolie lors des travaux de reconstruction du transept et du chœur (commencés dans le dernier tiers du XIV siècle $^{8}$ ). À l'occasion de cette reconstruction, la dernière travée orientale de la nef gothique fut modifiée et raccourcie, afin de réaliser les piles occidentales de la croisée du transept. D'autres travaux vinrent compléter ce vaste ensemble cathédral. Une chapelle commandée

2. Couffon, René, "Un catalogue... ", art. cit., p. 51.

3. Ibid., p. 53.

4. Ibid., p. 55 .

5. Continuation de la Chronique de Richard Lescot, religieux de Saint-Denis, Paris, éd. Jean le Moine, Société de l'Histoire de France, 1896, p. 68.

6. CoRnon, Raymond, " La cathédrale de Tréguier... ", art. cit., p. 104.

7. On date la construction de cette tour du XII ${ }^{\mathrm{e}}$ siècle. Ibid., p. 103.

8. Le blason ornant la clef de voûte de la deuxième travée du chœur est celle de l'évêque Morelli (1385-1400). 
par le duc Jean $\mathrm{V}$ afin d'accueillir son tombeau fut ajoutée au nord des trois travées les plus orientales de la nef à partir de 1442 (fig. 1), ce qui amena la destruction d'une partie des murs des bas-côtés ${ }^{9}$; puis, dans la deuxième moitié du $\mathrm{XV}^{\mathrm{e}}$ siècle, eut lieu la construction du vaste cloître au nord du chœur gothique ${ }^{10}$. Ainsi, si la tour Hasting est un vestige de l'ancienne cathédrale romane, la nef qui nous intéresse aujourd'hui est la partie de l'église par laquelle on inaugura le nouvel édifice gothique, ainsi qu'un nouveau parti gothique, comme nous le verrons. Cette nef ${ }^{11}$ à trois vaisseaux, réalisée dans un granite local, s'étend sur sept travées irrégulières et se trouve fermée à l'ouest par un simple mur droit. Deux portails, abrités par des porches monumentaux, donnent accès à l'intérieur de la nef. L'un est placé au centre de la façade occidentale, et l'autre ouvre sur la cinquième travée du bas-côté sud. L'élévation du vaisseau central est à trois niveaux et l'ensemble est coiffé par des voûtes d'ogives quadripartites qui culminent à 18 mètres dans le vaisseau central.

Cette partie de l'édifice fut visiblement commencée par l'ouest, et semble avoir été réalisée au moins en deux temps, deux partis différents étant repérables au premier niveau de la nef. Ainsi, le premier niveau de la façade, son portail abrité d'un vaste porche, et les trois travées les plus occidentales de la nef, furent réalisés dans un premier temps. Plusieurs indices confirment cette hypothèse.

9. LEguAY, Jean-Pierre, Un réseau urbain au Moyen Âge, les villes du duché de Bretagne aux XIV et $X V^{e}$ siècles, Paris, Maloine, 1981, p. 192.

10. Droguet, Alain, "Les comptes de la fabrique de la cathédrale de Tréguier (14801542) ", Artistes, artisans et production artistique en Bretagne au Moyen Âge, X. Barral i Altet et al. (éd.), Rennes, 1983, p. 209.

11. D’après les dossiers conservés à la Médiathèque du Patrimoine (Paris), les travaux de restauration n'ont jamais été de grande ampleur dans la nef. Les architectes rendent compte de projets de restauration pour la couverture dès 1841; une série de pièces de bois pour la charpente a ensuite été remplacée. Par ailleurs, on déboucha 14 fenêtres qui avaient été maçonnées et on remplaça les meneaux des remplages, ainsi qu'un grand nombre de vitraux. 
Figure 2 - Tréguier, cathédrale Saint-Tugdual, piles du bas-côté nord de la nef, vers l'ouest

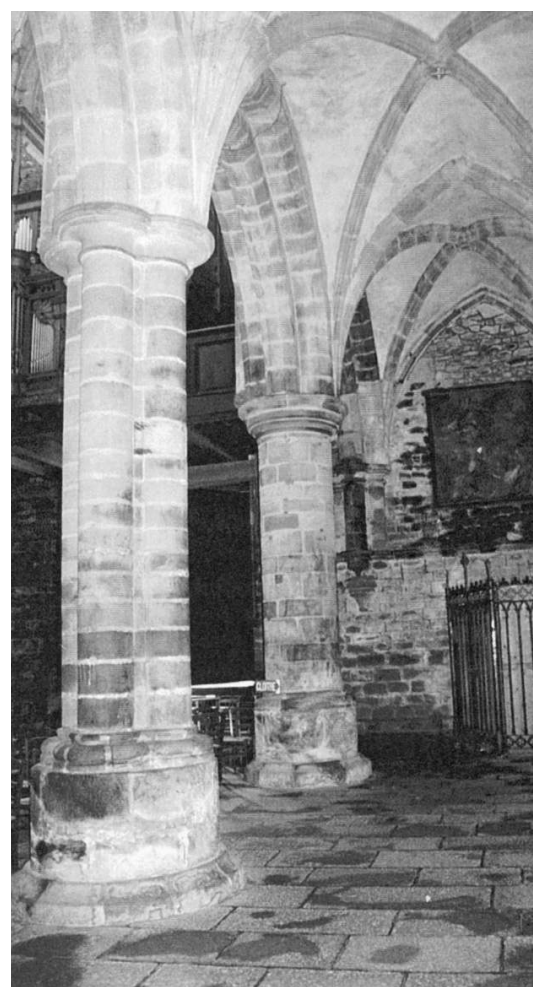

Figure 3 - Tréguier, cathédrale Saint-Tugdual, piles du bas-côté sud de la nef, vers l'est

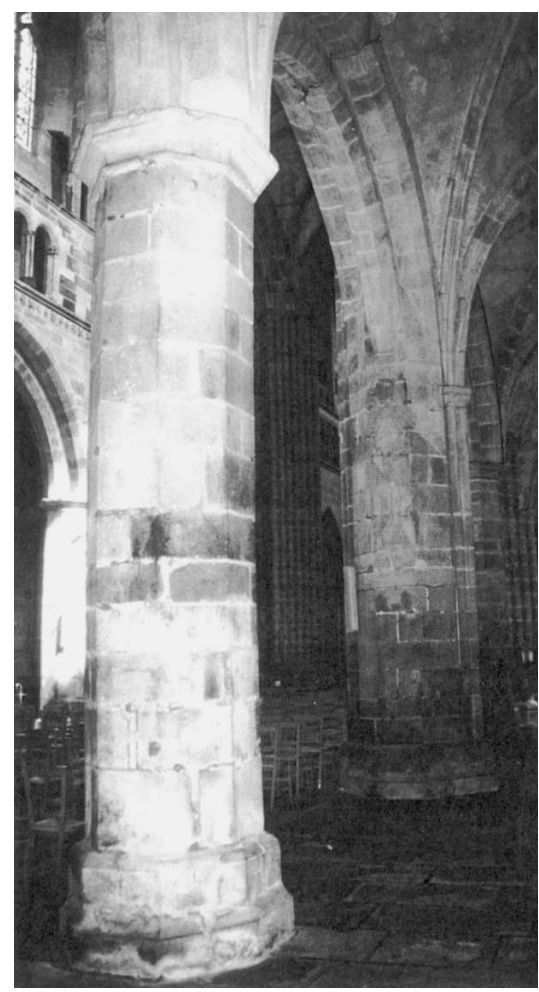

Un premier indice ressort de l'observation des piles. Celles des grandes arcades des trois travées les plus occidentales adoptent un rythme alterné, composé de piles monocylindriques et de piles à quatre colonnes fortement saillantes et engagées dans un mince noyau qui n'apparaît qu'à peine (fig. 2). Au-delà de ces premières piles, à peu près identiques au nord et au sud, ces supports sont remplacés soit par des piles composées d'un fort noyau polygonal dans lequel quelques minces colonnettes sont engagées, soit par de simples colonnes au fût polygonal (fig. 3). Alors qu'au sud, un semblant d'alternance fut conservé, au nord, l'alternance fut abandonnée et trois colonnes polygonales se succèdent après une première pile composée. Il y eut donc ici un changement de parti bien visible.

Un second indice ressort de l'observation du décor. Au cours des premiers travaux, un caractère particulier avait été donné aux premières travées de la nef puisque les corbeilles des piles du vaisseau central étaient parfaitement lisses, alors que celles des bas-côtés recevaient un décor sculpté; cette particularité ne fut pas respectée par la suite. D’autres détails furent modifiés : les premières colonnes engagées dans les murs des bas- 
côtés sont fortes et uniques, possèdent des tailloirs circulaires, et reposent sur des culots. Au cours de la campagne suivante, le parti fut modifié, puisque, au nord, les mêmes supports sur culots apparaissent, mais leurs tailloirs sont polygonaux et plus plats; au sud, la transformation est plus franche, ces supports ayant été remplacés par de triples colonnettes, très minces et beaucoup plus élancées puisqu'elles naissent à la base des murs latéraux.

Ainsi, après l'édification du portail et des premières travées occidentales de la nef, un arrêt de la construction semble avoir eu lieu, d'une durée indéterminée. Par la suite, les travaux se sont probablement poursuivis dans le bas-côté nord, où le premier parti a été à peu près respecté, et, plus tard, dans le bas-côté sud; en revanche, les grandes arcades ont sans doute été construites d'abord au sud, où l'on retrouve l'alternance des premières travées, puis au nord, où le rythme alterné est rompu et définitivement abandonné. Au cours de cette deuxième campagne de travaux, l'architecte fit preuve d'hésitations et l'œuvre réalisée est moins homogène, les travées adoptant des largeurs différentes les unes des autres, et aucune des piles composées n'ayant été réalisée sur le même modèle ${ }^{12}$.

Une évolution dans les choix formels des arcs des grandes arcades et des voûtes des bas-côtés est également perceptible. La réalisation des arcs commença au sud-ouest, où, dans les trois premières travées, ils sont en calcaire et profilés de trois rouleaux aux angles creusés de minces cavets. Ailleurs dans la nef, les rouleaux sont en granite, beaucoup plus sobres, et seulement composés de deux rouleaux chanfreinés. De même, les trois premières voûtes au sud-ouest ont été montées avant les autres : les arcs et les nervures sont en calcaire, de forme torique et dégagés par des cavets latéraux, alors que partout ailleurs, ils sont en granite, et adoptent un profil en amande souligné d'un listel.

La chronologie des parties hautes paraît moins complexe (fig. 4). Une fois le premier niveau réalisé, les portails et les porches construits, les travaux se poursuivirent par la frise de calcaire qui souligne le triforium, le niveau du triforium, les fenêtres hautes, ainsi que la grande baie présente dans la façade occidentale. Ces parties hautes furent conçues dans le respect des choix adoptés au premier niveau. Par exemple, les ébrasements des fenêtres hautes sont aussi prononcés que ceux des fenêtres des bascôtés, et le réseau de la grande verrière occidentale évoque celui qui orne le porche sud; de même, les triples colonnettes du mur sud-est des bascôtés furent reprises de manière à scander régulièrement les travées de l'élévation. Enfin, les cadres rectangulaires typiquement rayonnants qui cernent les arcatures du triforium, trouvent leur écho dans les murs latéraux du porche méridional.

Dans l'ensemble, les niveaux supérieurs sont plus homogènes que les parties basses, bien que quelques variantes soient visibles; en particulier,

12. En particulier, les colonnes engagées qui sont, selon les cas, soit uniques, soit triples. 
Figure 4 - Tréguier, cathédrale SaintTugdual, élévation nord de la nef

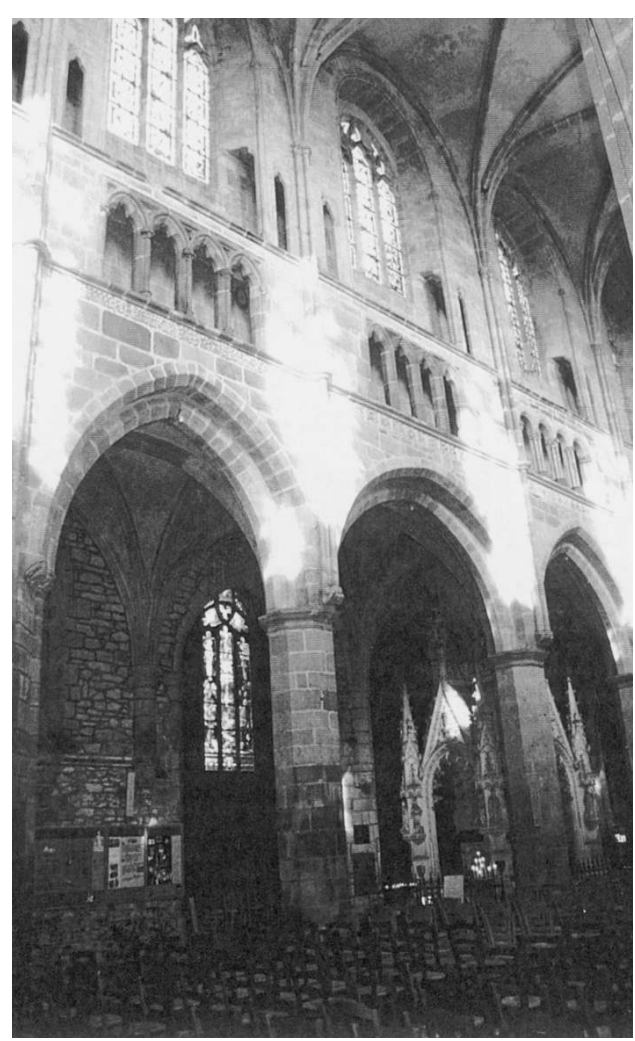

les deux dernières travées nord-est du triforium diffèrent des autres, le matériau utilisé étant le calcaire, les arcs adoptant une forme en plein cintre, et les piles étant exceptionnellement composées de faisceaux de colonnettes. Par ailleurs, les autres travées du triforium ne sont pas parfaitement identiques, les arcatures étant soit chanfreinées, soit moulurées, et pourvues ou non de chapiteaux sculptés. Toutes ces différences, parfois subtiles, sont difficiles à interpréter, et ne nous fournissent aucun renseignement sur la marche des travaux de ce niveau. Elles semblent plutôt indiquer que, comme ce fut le cas pour les parties basses, les choix formels étaient opérés au fur et à mesure de l'avancée des travaux, et que différentes formules étaient expérimentées successivement par les responsables de la construction. Cette méthode obligeait l'architecte à être en permanence sur le chantier, afin d'orienter la construction et de faire tailler les blocs de pierre en fonction de ses décisions et de ses choix.

\section{Définition du parti architectural de la nef de Tréguier}

\section{La silhouette (fig. 5)}

Plutôt sobre depuis l'extérieur, la silhouette de la nef de Tréguier est empreinte d'une forte horizontalité, à peine contrebalancée par les verticales des culées et des pinacles des arcs-boutants à simple volée. Seuls les remplages des fenêtres et des entrées des porches, ainsi que les balustrades, viennent animer à l'extérieur cet austère bâtiment de granite. La façade est des plus simples (fig. 6). Il s'agit d'un mur qui ferme les trois vaisseaux de la nef à l'ouest. Au sud du portail central, une tourelle de plan carré permet d'accéder aux escaliers et aux couloirs menant aux niveaux supérieurs; au nord, un contrefort massif surmonté d'une forte culée et d'un pinacle lui fait pendant. Au-dessus du portail prend place une immense baie gar- 
nie de remplages, et celle-ci se trouve flanquée de deux tourelles d'escaliers de plan carré, couronnées par des lanternons.

Figure 5 - Tréguier, cathédrale Saint-Tugdual, flanc sud de la nef

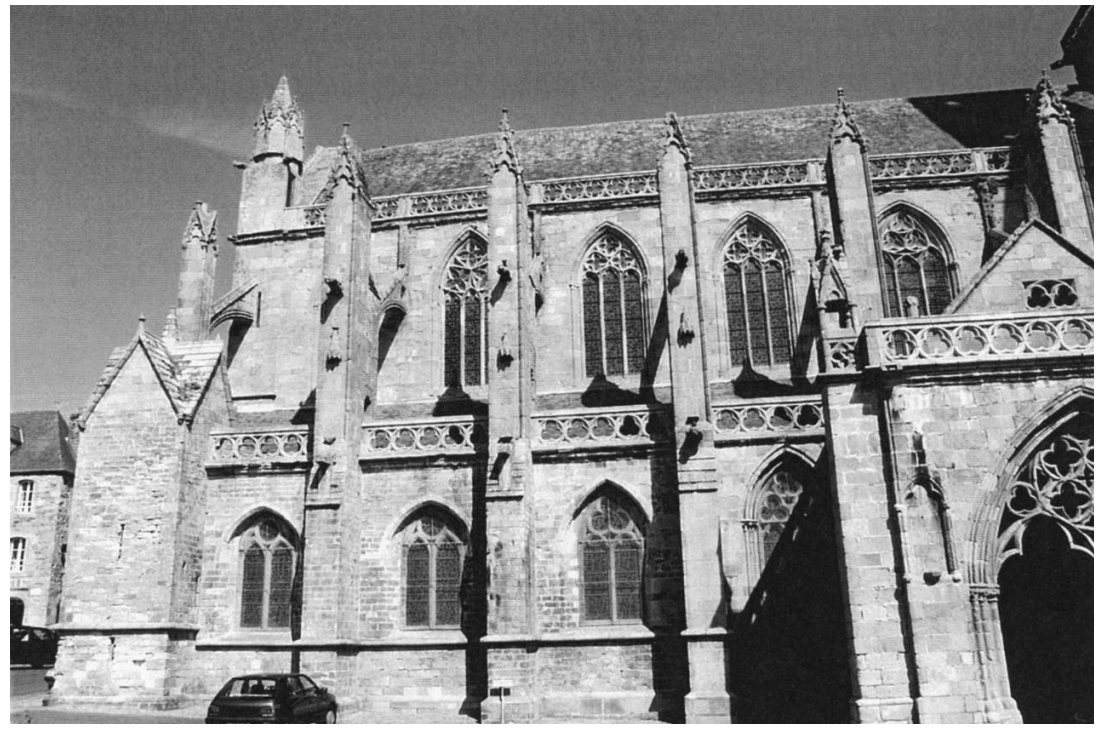

Figure 6 - Tréguier, cathédrale Saint-Tugdual, façade occidentale de la nef et son porche

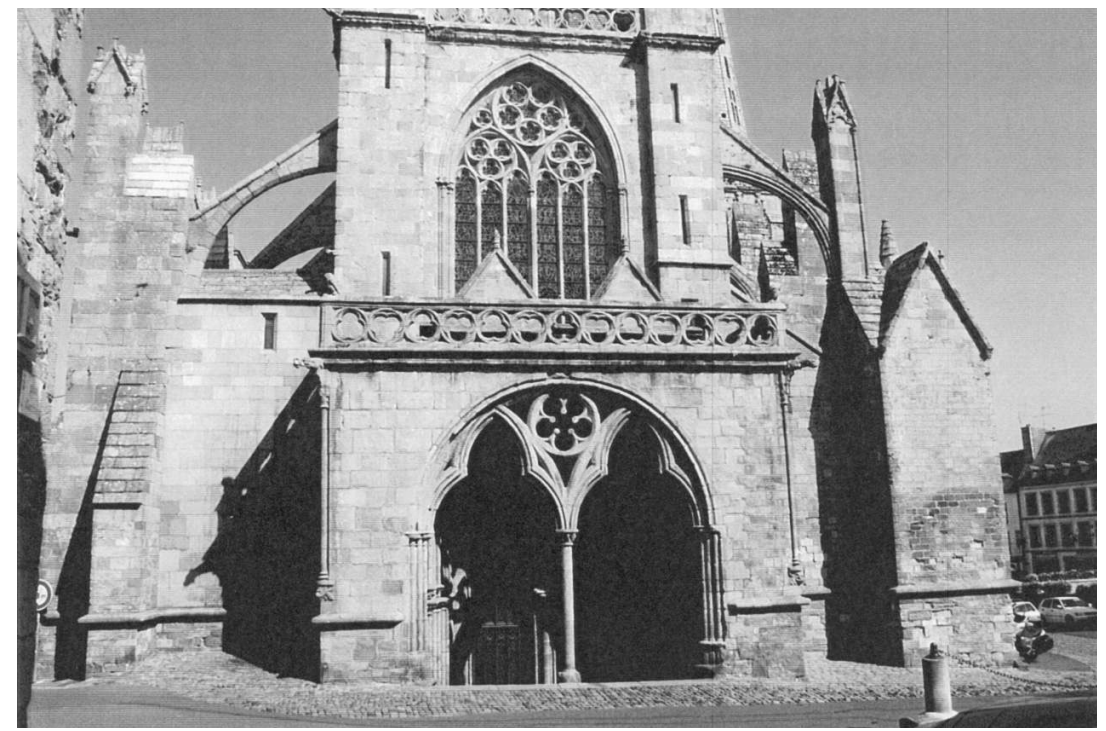


Cette composition très sobre et un peu archaïque, avec ses tourelles carrées, ne peut manquer d'évoquer quelques façades romanes de Normandie, comme celle de Saint-Georges-de-Boscherville, dont le parti se retrouve à quelques reprises dans l'ouest de l'Angleterre, comme à Tewkesbury, par exemple. À Tréguier, cependant, la façade est rendue plus monumentale par la présence d'un porche très saillant, absent de ces façades romanes, et la grande baie rayonnante appartient bien à son époque.

\section{Les portails et les porches}

Figure 7 - Tréguier, cathédrale Saint-Tugdual, porche occidental de la nef

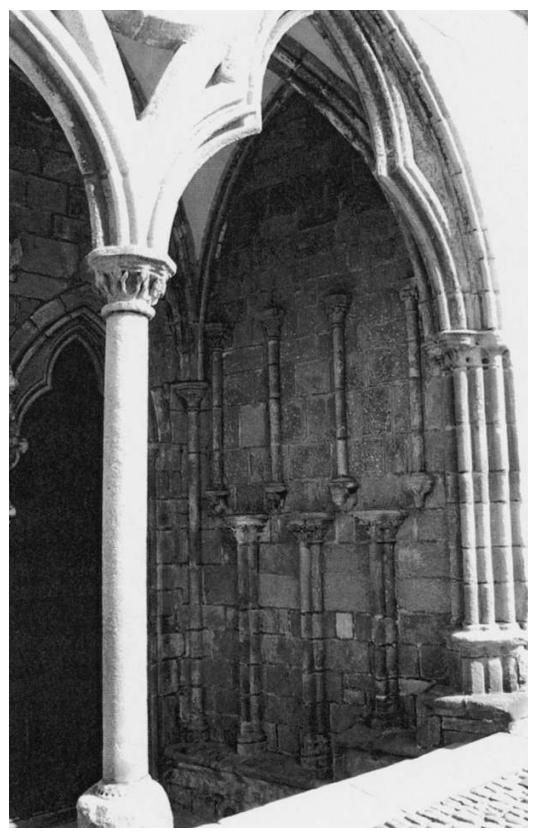

Figure 8 - Tréguier, cathédrale Saint-Tugdual, porche méridional de la nef

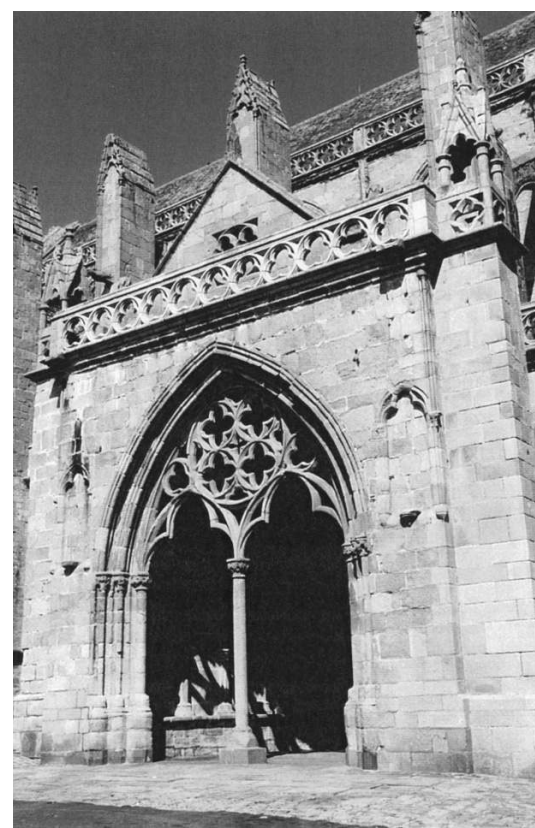

Il est important d'évoquer rapidement les deux portails de la nef et les vastes porches qui les abritent. Très monumentaux et très soignés, ils viennent enrichir la silhouette extérieure de l'édifice, qui, par ailleurs, est des plus sobres. Le décor y est plus recherché au sud ${ }^{13}$ qu'à l'ouest, indiquant d'emblée que la façade principale de la cathédrale est la façade méridionale, celle qui est tournée vers le centre ville ${ }^{14}$.

13. Les documents écrits et figurés du XIX ${ }^{\mathrm{e}}$ siècle conservés à la Médiathèque du Patrimoine montrent que le porche méridional avait été transformé en chapelle et que l'entrée avait été bouchée, le remplage étant resté, cependant, intact.

14. Le grand portail du bras sud du transept, réalisé à la fin du Moyen Âge, ainsi que l'immense verrière qui le surmonte, corroborent cette affirmation. 
Figure 9 - Tréguier, cathédrale Saint-Tugdual, mur ouest du porche méridional de la nef

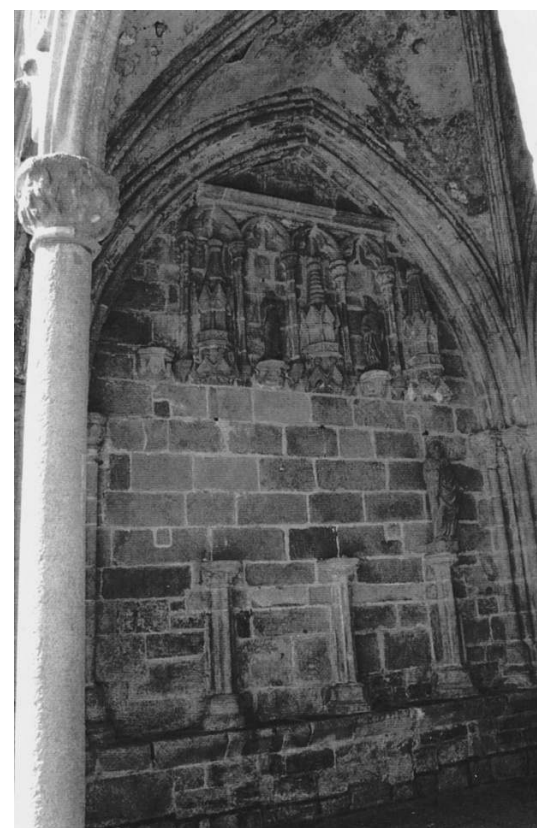

Figure 10 - Tréguier, cathédrale Saint-Tugdual, portail méridional de la nef

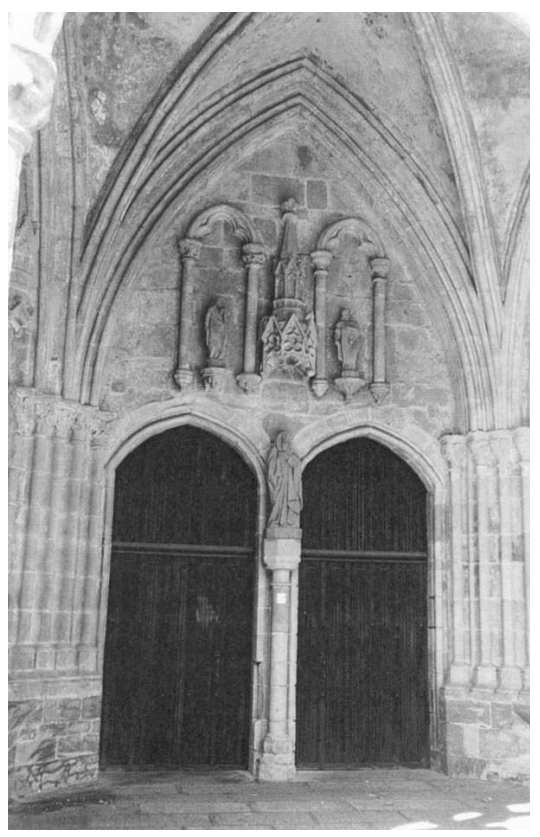

Les deux portails ont pourtant beaucoup en commun. Les deux entrées sont séparées par un trumeau au devant duquel une colonne est destinée à accueillir une statue en ronde-bosse, celle-ci étant couronnée par un dais engagé dans le tympan appareillé. Les entrées du portail occidental sont enrichies par des colonnettes à chapiteaux qui les flanquent, et des arcatures trilobées reposant sur des marmousets, décor absent au sud. En revanche, le tympan méridional est bien plus décoré (fig. 10), le dais étant plus important et plus richement sculpté, et de part et d'autre de ce dais, deux statuettes prenaient place sur de petits culots servant de socles . Ces petites statues étaient placées dans des niches constituées de simples colonnettes coiffées par des arcs à trilobes inscrits, le tout engagé dans le tympan appareillé.

Les deux porches ${ }^{15}$, très profonds, sont coiffés de voûtes sur croisées d'ogives qui reposent sur des murs latéraux maçonnés. Les deux entrées de ces porches sont mises en valeur par la présence de remplages monumentaux créant, dans chacun des cas, deux entrées séparées par une colonne en délit, faisant écho au parti des portails. Les remplages sont constitués de deux grands arcs à trilobes inscrits surmontés par un oculus. À l'ouest (fig. 6), les trilobes sont très étirés et l'oculus, de petites

15. Les dispositions sont originales à l'ouest puisque la voûte du porche occidental couvre une volée de marches, la nef étant située en contrebas. 
dimensions, est occupé par un motif polylobé. Au sud (fig. 8), la composition est plus soignée; les arcs sont moins allongés et l'oculus, plus monumental, accueille quatre quadrilobes inscrits, composition qui évoque le remplage de la grande verrière occidentale. Enfin, les murs latéraux des porches étaient à l'origine, dans les deux cas, occupés par des statues dont seuls les supports et les niches subsistent -, engagés dans la maçonnerie; à l'ouest, des faisceaux de colonnettes, disposés en quinconce sur deux niveaux, supportaient et flanquaient trois statues sur chaque paroi (fig. 7). Au sud, trois statues prenaient également place sur trois faisceaux de colonnettes (fig. 9); mais la composition y est rendue plus complexe par la présence de petites niches composées de colonnettes et d'arcs trilobés, qui abritent alternativement des dais sculptés et des socles placés à la même hauteur, ces derniers étant destinés à soutenir de petites statues ${ }^{16}$.

Ces porches et portails très amples, dont les décors sculptés et particulièrement recherchés tranchent avec la sobriété du parti de la nef, confèrent à cette cathédrale un caractère à la fois soigné et monumental tout à fait particulier.

\section{Le vaisseau central}

Malgré les tâtonnements, les changements de parti et les reprises, l'élévation principale de la nef de Tréguier est homogène et présente un parti général qu'il faut maintenant définir (fig. 11). Nous avons affaire à une élévation à trois niveaux aux proportions peu élancées et à la respiration ample.

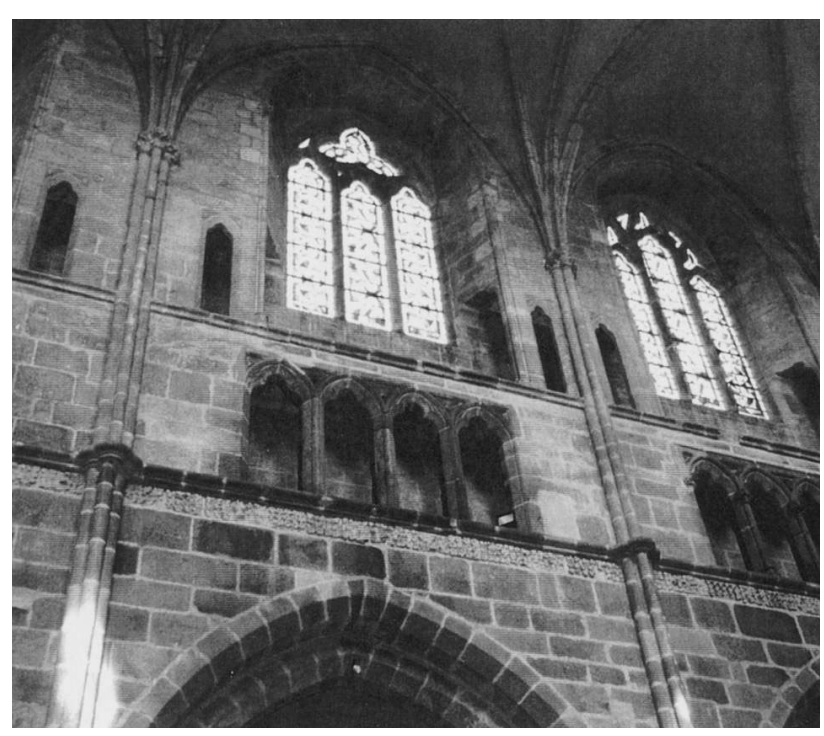

Figure 11 Tréguier, cathédrale SaintTugdual, mur gouttereau nord de la nef

16. Ces compositions sont centrées de manière approximative, et un socle sur le mur ouest, un dais et un socle sur le mur est, ne sont pas pris dans ce cadre. 
Les grandes arcades, larges et dont l'arc est à peine brisé, sont sobres et irrégulières. La variété des piles, qui n'ont en commun que leur hauteur peu importante, entraîne une diversité dans le rythme général de l'élévation. En effet, dans la plupart des cas, les colonnes de fond naissent sur des culots placés au-dessus des chapiteaux des piles (fig. 4). En revanche, les quelques piles composées réalisées au cours de la deuxième campagne de construction accueillent des faisceaux de triples colonnettes qui s'élancent sur toute la hauteur du vaisseau, depuis le sol jusqu'à la retombée des voûtes. La scansion verticale n'est donc insistante que dans ces travées.

Au sein de chaque travée, chacun des niveaux est clairement séparé des autres par la présence de bandeaux sculptés ou moulurés qui ne sont pas sans évoquer l'architecture normande. La séparation entre grandes arcades et triforium est très nette, puisque, à une frise de calcaire sculptée, s'ajoute une moulure qui se poursuit sur les colonnes de fond sous forme de bagues. Les deux niveaux supérieurs sont plus étroitement réunis : sous les fenêtres court une moulure beaucoup moins ourlée que la précédente qui, elle, est régulièrement interrompue par les colonnes de fond.

Sans revenir trop longuement sur le premier niveau de l'élévation, il est possible de souligner le caractère plein et rond des supports alternés des travées occidentales, qui tranche avec la très grande sécheresse des supports orientaux de la seconde campagne de travaux. Les socles et tailloirs circulaires des piles de l'ouest laissent la place à des formes polygonales, les moulures toriques à des profils plus acérés et simplifiés. Toutes ces caractéristiques, ainsi que la simplicité des corbeilles lisses et courtes de l'ensemble des piles de la nef, et la sobriété des rouleaux des arcs, ne sont pas sans évoquer l'architecture gothique anglaise. Sans parler du changement de parti des piles, probablement lié à un changement de maître, la variété des supports et des rythmes de la nef peut être liée à un goût certain pour les piles complexes et plastiques, qui ne peut manquer, lui-aussi, de rappeler l'architecture gothique anglaise ${ }^{17}$. Il serait également intéressant de rechercher des analogies, qui existent très certainement, avec l'architecture française de la fin du XIII ${ }^{\mathrm{e}}$ siècle et des premières années du siècle suivant ${ }^{18}$.

Le niveau du triforium est plus uniforme et plus caractéristique. Il s'agit d'un véritable niveau intermédiaire, très court, entièrement aveugle, et composé d'arcatures de hauteur identique, dont le parti général très simple est dérivé des triforiums apparus en France dans les édifices gothiques comme Soissons ou Chartres. Quelques détails caractérisent cependant ce triforium et témoignent de l'époque tardive de sa réalisation. En effet, ce n'est pas un triforium continu, ses arcatures étant regroupées par quatre ${ }^{19}$ à peu

17. Ce sujet est évoqué par Raymond Connon, "La cathédrale de Tréguier... ", art. cit., p. 115, 117-118, et par Roger BARRIE, "La cathédrale Saint-Yves... ", art. cit., p. 226-294.

18. BonY, Jean, French Gothic Architecture of the $12^{\text {th }}$ and $13^{\text {th }}$ Centuries, Berkeley 1983, p. 429 et suivantes.

19. Sauf dans la dernière travée orientale, raccourcie lors de la construction du transept, où l'on trouve seulement trois arcatures. 
près au centre de chaque travée, chaque groupe étant flanqué de part et d'autre d'une importante surface murale. La largeur de ces groupes d'arcatures est équivalente à celle de la fenêtre haute placée au-dessus, ce qui contribue à unifier ces deux niveaux. Ces arcatures sont très étroitement circonscrites par un cadre rectangulaire ${ }^{20}$, motif courant dans les églises rayonnantes et très répandu dès le dernier tiers du XIII ${ }^{\mathrm{e}}$ siècle. Cependant, ce cadre rythme un niveau très court, aveugle, où le réseau rayonnant n'a pas sa place; là encore, il est important de se tourner vers l'ouest de l'Angleterre, où ce type de triforium apparaît assez fréquemment à la fin du XIII ${ }^{\mathrm{e}}$ siècle et au siècle suivant, à Chester ou à Exeter ${ }^{21}$ par exemple.

Les fenêtres hautes de la nef de Tréguier, peu élancées, n'occupent pas toute la surface disponible dans chaque travée. Elles sont creusées dans un mur très présent et très épais, et sont ébrasées vers l'intérieur, le remplage étant rejeté vers l'extérieur. Ces ébrasements laissent visibles les entrées de la galerie qui dédouble le mur épais de ce niveau et qui se superpose harmonieusement à celle du triforium. Ces fenêtres sont ornées d'une simple arcature à chapiteaux sculptés, à l'intérieur et à l'extérieur. La sobriété est de règle puisque tous les remplages sont dépourvus de moulures, bien que leurs dessins varient d'une travée à l'autre ${ }^{22}$. De part et d'autre de ces fenêtres, deux minuscules arcatures trilobées, prises sous un cadre rectangulaire, viennent éclairer cette galerie ${ }^{23}$.

Nous sommes en présence d'un édifice à mur épais dont le parti est issu de l'architecture romane anglo-normande, parti qui fut conservé au XIII ${ }^{\mathrm{e}}$ siècle et aux siècles suivants en Angleterre et dans certaines régions françaises comme la Normandie, la Bretagne ou la Bourgogne. Les petites arcatures latérales qui percent le mur de part et d'autre de la fenêtre sont issues de la même famille architecturale. Il s'agit d'un motif dérivé des triples arcatures de type loggia, apparues à Winchester au XI ${ }^{\mathrm{e}}$ siècle et à la Trinité de Caen ${ }^{24}$. Bien que présent dans quelques édifices normands, ce motif connut très peu de succès sur le continent alors qu'il est des plus courants en Angleterre, tant dans les églises romanes que dans les édifices gothiques. Mais l'architecte de Tréguier ne se contenta pas de plagier une œuvre gothique anglaise. Le parti choisi est original puisqu'il combine le parti de la simple fenêtre à ébrasements profonds et celui de la fenêtre de type log-

20. Les arcatures des deux travées nord-est, en calcaire et différentes des autres, ne possèdent pas de cadre rectangulaire.

21. Jansen, Virginia, "Superposed Wall Passages and the Triforium Elevation of St. Werburg's, Chester ", Journal of the Society of Architectural Historians, XXXVIII/3, 1979, p. 223-243, et du même auteur, "The Design and Building Sequence of the Eastern Arm of Exeter Cathedral c. 1270-1310, A Qualified Study ", British Archaeological Association, Conference Transaction, Medieval Art and Architecture at Exeter Cathedral, XI, 1991, p. 34-56.

22. Quelques fenêtres ont été débouchées au XIX ${ }^{\mathrm{e}}$ siècle, mais il ne semble pas que les remplages aient été remplacés dans leur totalité.

23. Ces minuscules arcatures ont disparu à l'extrémité orientale de la nef, probablement lors de la reconstruction du transept.

24. BAYLE, Maylis, La Trinité de Caen, sa place dans l'histoire de l'architecture et du décor roman, Paris/Genève, 1979, p. 59-61. 
gia. Par ailleurs, les arcatures latérales sont très atrophiées par rapport au modèle traditionnel. Il s'agit donc d'une évocation originale et tardive de ce parti, modernisé par la présence de remplages variés.

Dans le mur de la façade occidentale (fig. 6), une immense baie garnie de réseaux rayonnants éclaire le vaisseau central ${ }^{25}$. Elle évide le mur sur une hauteur équivalant à celle du triforium et des fenêtres hautes. Cependant, elle n'occupe pas toute la surface de mur disponible. Là encore, le remplage est rayonnant ${ }^{26}$, ce qui n'est pas le cas du traitement du mur, encore très présent et très épais. Par ailleurs, cette baie est fortement ébrasée, les entrées de la galerie passant au-devant étant bien visibles. Cette fenêtre à remplages située à l'extrémité d'un vaisseau par ailleurs très peu évidé est également un trait évoquant l'Angleterre gothique ${ }^{27}$.

L'élévation de l'ancienne cathédrale de Tréguier est atypique en France, en ce $\mathrm{XIV}^{\mathrm{e}}$ siècle, pour plusieurs raisons. La hauteur sous voûtes est faible, l'élan vertical est très peu affirmé, et les différents partis choisis sont, à plus d'un titre, archaïques par rapport à l'architecture française contemporaine. Lorsque le style rayonnant apparaît, ce qui est le cas à maintes reprises dans la nef de Tréguier, c'est visiblement à travers le prisme de l'interprétation anglaise.

\section{Une architecture gothique bretonne}

Quelle que soit l'origine de cette architecture, elle n'est pas unique en Bretagne, et les grands traits évoqués ici se retrouvent non seulement dans certaines cathédrales, mais aussi dans quelques églises plus modestes. La plupart des cathédrales des XIV et $\mathrm{XV}^{\mathrm{e}}$ siècles présentent des silhouettes longues et basses (les hauteurs sous voûtes variant de 15 à 22 mètres environ), des ouvertures plutôt modestes, et des arcs-boutants réduits à leur plus simple expression. Leurs élévations ont en commun des proportions généreuses, des triples niveaux avec triforiums courts et aveugles, et des fenêtres hautes plutôt réduites mais garnies de remplages rayonnants. Les murs sont épais et traversés par des galeries au dernier niveau, qui se superposent à celles des triforiums.

Dès le troisième quart du XIII ${ }^{\mathrm{e}}$ siècle, la nef de la cathédrale de Dol $^{28}$ (fig. 12) annonce certains partis présents à Tréguier : c'est le cas de la sobriété des modénatures des arcs des grandes arcades, et de la présence d'une immense fenêtre percée dans la façade occidentale. D'ailleurs cette fenêtre, modifiée à la période moderne, était certainement garnie de rem-

25. Les formes étirées de ses réseaux, les profils acérés des moulures appartiennent bien à leur temps, et se retrouvent fréquemment en Angleterre, là aussi associés à des traits évoquant l'architecture romane.

26. Cette baie est plus ornée que les autres : les arcatures moulurées sont doublées, et le réseau principal est décoré de colonnettes à chapiteaux et de moulures.

27. Le Boulc'H, Anne-Claude, La cathédrale de Dol, Rennes, PUR, 1999, p. 126.

28. Ibid. 
Figure 12 - Dol, cathédrale SaintSamson, élévation méridionale de la nef

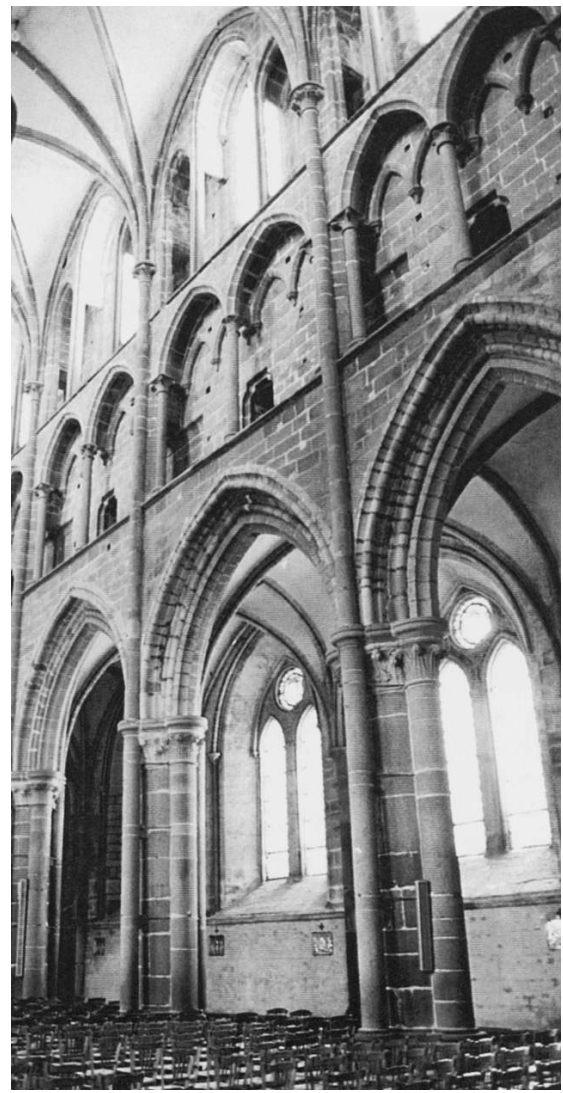

plages à la manière de celle de Tréguier ${ }^{29}$. À Dol, le mur n'est pas, là non plus, parfaitement évidé. Le triforium aveugle est lui aussi surmonté de fenêtres hautes percées dans un mur épais, et enrichi des trois arcatures issues du modèle roman. Mais ce motif est plus développé à Dol, les arcatures latérales étant plus élancées et formant un véritable rythme ternaire, qui a été fortement atténué à Tréguier. Le chœur et le transept de Dol, un peu plus tardifs, reprennent à peu près ce parti; les grandes verrières garnies de remplages rayonnants qui occupent les extrémités des bras du transept et du chœur, avec leurs couronnements de grands oculi garnis de petits motifs polylobés, ne sont pas sans évoquer la verrière occidentale de Tréguier. Enfin, le portail du bras sud du transept de Dol (fig. 13) annonce les portails trégorrois. On retrouve en particulier les deux entrées trilobées, la colonne engagée dans le trumeau qui supportait une sculpture en ronde-bosse, le tympan maçonné dans lequel sont engagés deux socles latéraux qui accueillaient des statues. Une variante existe cependant ici, puisque le dais présent à Dol sert également de socle pour une statue supplémentaire qui surmontait celle du trumeau.

L'œuvre la plus proche de la nef de Tréguier est celle de Saint-Pol-deLéon $^{30}$ (fig. 14). Cette cathédrale a, elle aussi, été réalisée en plusieurs temps, la nouvelle nef gothique ayant été construite à l'ouest d'un chœur roman. Bien qu'un peu moins élancées ${ }^{31}$, les proportions générales et la silhouette y sont à peu près les mêmes qu'à Tréguier et à Dol; mais ici, l'intérieur de l'édifice est entièrement en calcaire importé de Normandie, alors que l'enveloppe extérieure est constituée d'un appareil de granite. Plus homogène

\section{Ibid. p. 65 .}

30. LeCUREuX, Lucien, "Saint-Pol-de-Léon, cathédrale ", Congrès Archéologique de France, $81^{\mathrm{e}}$ session (Brest et Vannes), 1914, p. 82-93.

31. La hauteur sous voûte n'est que de 15 mètres. 


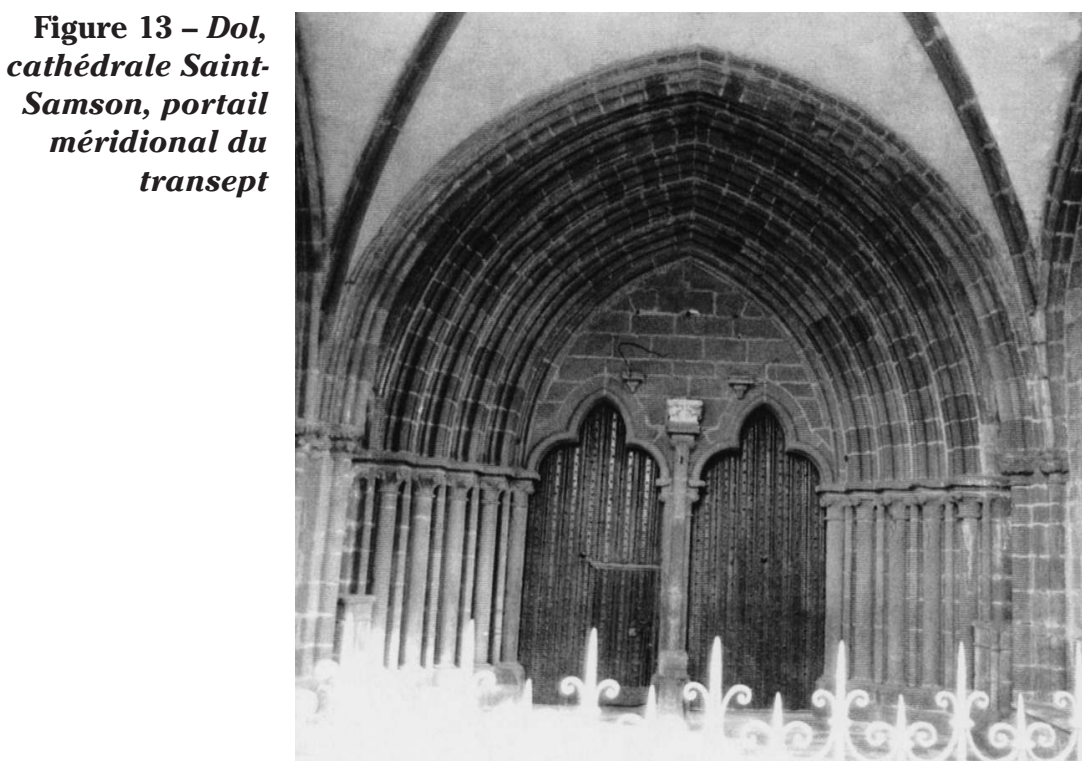

et édifié d'un seul jet, son vaisseau central est plus régulièrement scandé par des piliers uniformément composés et par des faisceaux de colonnettes qui unissent l'ensemble des trois niveaux. Comme à Tréguier, le triforium est court et aveugle. Il ne s'agit pas d'un triforium continu, non parce que les arcatures sont regroupées au centre de la travée comme à Tréguier, mais parce qu'un motif particulier individualise les travées : ce motif est constitué de deux arcatures latérales étroites, et de deux arcatures centrales plus amples et presque en plein cintre. Les supports de ces arcatures sont simplement chanfreinés, et les arcs pénètrent directement dans les supports, caractéristiques qu'on retrouve à plusieurs reprises dans la nef de Tréguier ${ }^{32}$. Enfin, dans ces deux nefs se retrouve la petite frise
Figure 14 - Saint-Pol-de-Léon, cathédrale, élévation nord de la nef

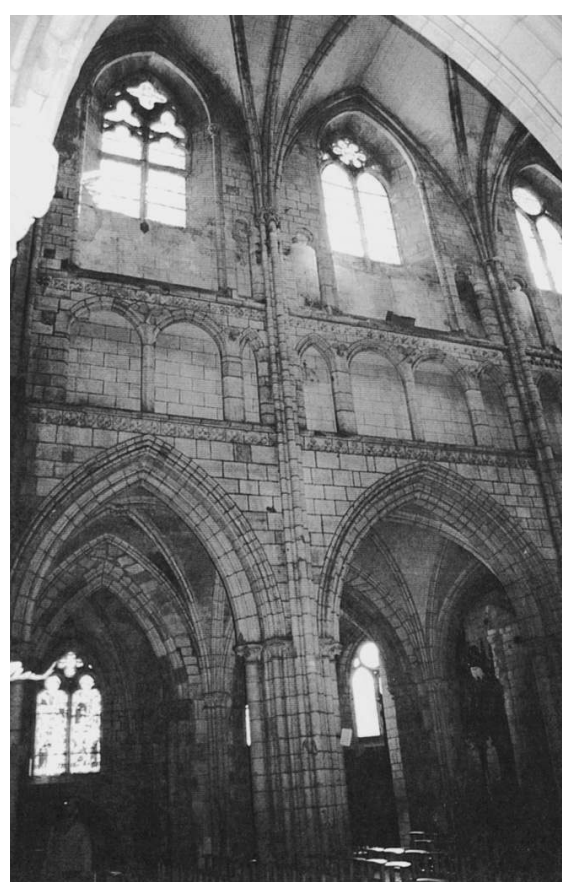

32. En particulier dans la pile composée sud-est des grandes arcades. 
Figure 15 - Saint-Pol-de-Léon, cathédrale, façade occidentale

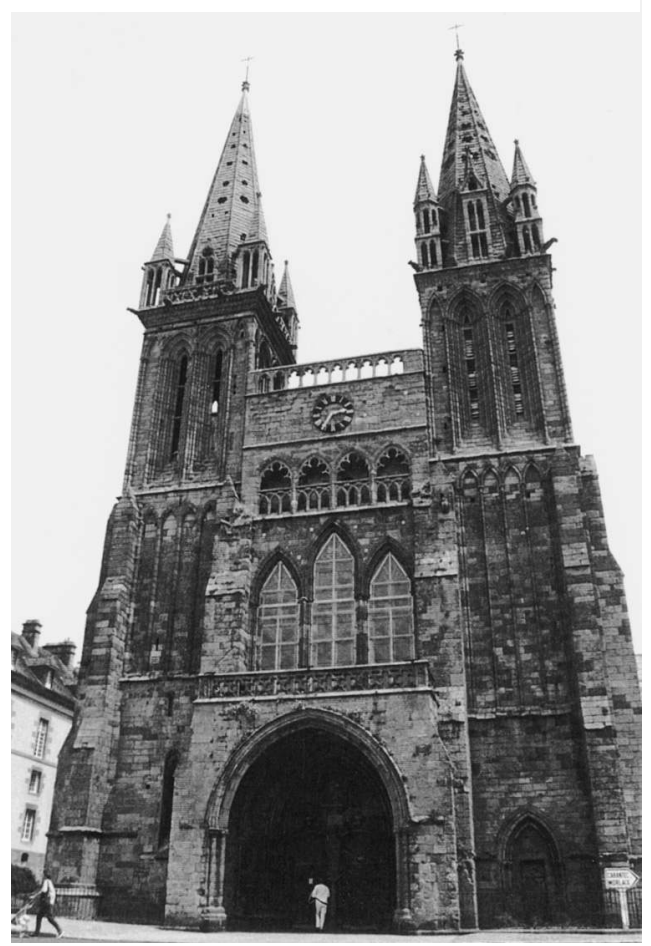

sculptée sous le triforium, frise qui apparaît aussi sous les fenêtres hautes de SaintPol.

Le dernier niveau est presque identique à celui de Tréguier, où l'on retrouve les fenêtres à remplages, les mêmes ébrasements, la galerie, et les arcatures latérales atrophiées. Les remplages y sont également très variés, bien que les motifs choisis soient plus simples. La façade occidentale (fig. 15) est plus proche de celle de Dol que de celle de Tréguier, avec ses deux tours latérales; en revanche, le portail occidental et son très ample porche (fig. 16) appartiennent à la même famille que ceux évoqués plus haut. Le parti général du porche est le même, en plus simple, puisque l'entrée

Figure 16 - Saint-Pol-de-Léon, cathédrale, portail occidental et son porche

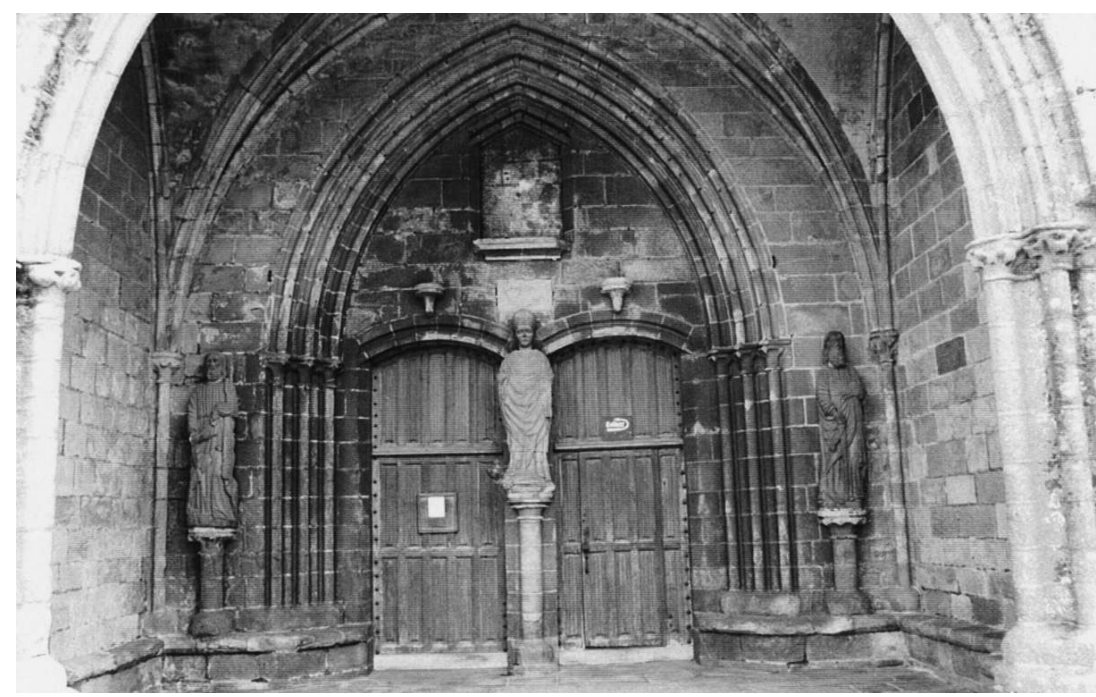


Figure 17 - Saint-Pol-de-Léon, cathédrale, portail méridional de la nef et son porche

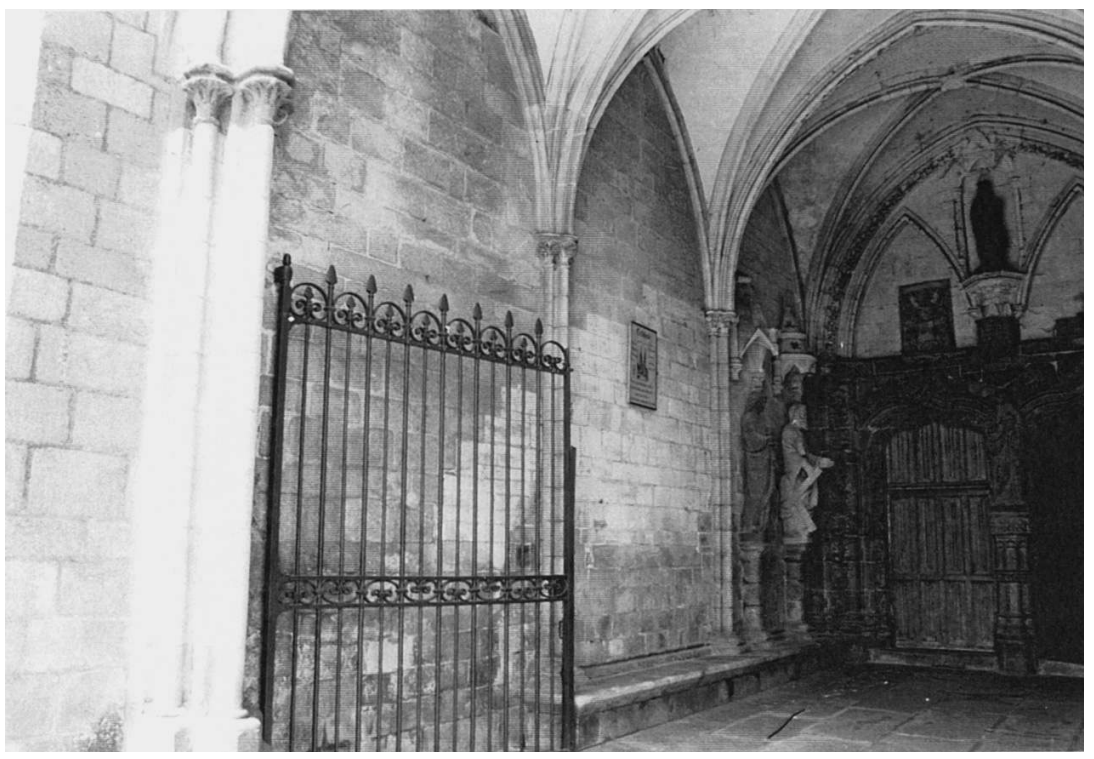

Figure 18 - Saint-Pol-de-Léon, cathédrale, tympan du portail méridional de la nef

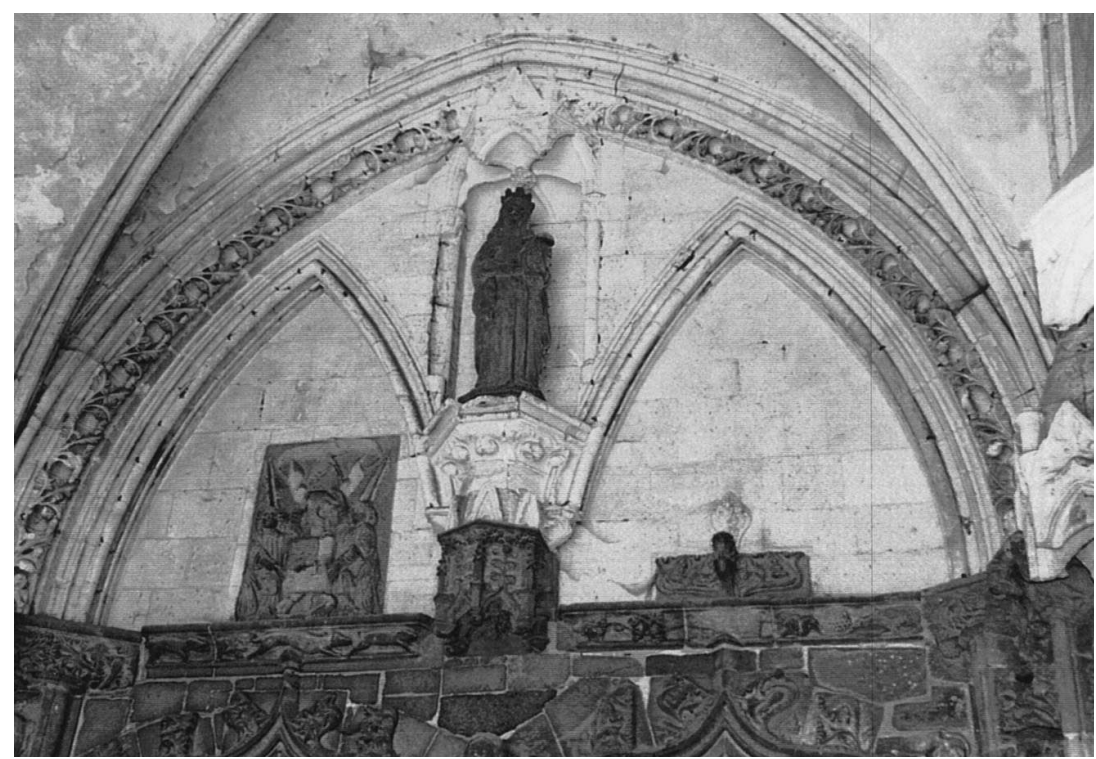


n'est constituée que d'une arcature dénuée de remplages. Le portail est, luiaussi, plus sobre, les entrées aux arcs surbaissés n'étant pas moulurées. Sur le tympan, le dais central de Tréguier a ici été remplacé par une petite niche peu profonde, aujourd'hui vide, elle-même flanquée des deux socles latéraux étroits qui recevaient des statuettes. Enfin, de part et d'autre des entrées, deux colonnes latérales répondent à la colonne du trumeau et accueillaient des statues monumentales.

Le portail méridional ouvrant sur le flanc de la nef de Saint-Pol, est, comme à Tréguier et à Dol, plus soigné que le portail occidental (fig. 17). Bien que fortement repris en sous-œuvre au $\mathrm{Xv}^{\mathrm{e}}$ siècle, l'agencement d'origine est encore visible. Seul subsiste le tympan de calcaire souligné par la grande frise sculptée de feuillages, et divisé en deux par des arcatures plaquées (fig. 18). Dans l'écoinçon séparant ces deux arcatures, une statue était placée dans une niche constituée de deux colonnettes surmontées d'un dais richement sculpté. Le socle de la niche sert également de dais pour la statue qui s'élevait, avant les remaniements, au-devant du trumeau. Enfin, il est probable que sous les deux petites arcatures plaquées du tympan, de part et d'autre de la statue centrale, des statuettes étaient fixées sur de petits socles; ceux-ci ont disparu, remplacés au Xve siècle par des éléments de granite. Le porche méridional (fig. 17) est beaucoup plus profond que tous ceux évoqués précédemment, puisqu'il s'étend sur quatre travées de plan barlong, voûtées d'ogives quadripartites. Ici encore, le calcaire a été associé au granite : les murs latéraux et les colonnettes engagées, ainsi que les nervures des voûtes, sont en granite, alors que les chapiteaux des colonnettes sont en calcaire. Seule la travée la plus proche du portail abrite des statues : sur chaque paroi, deux piles polygonales de granite reçoivent des statues monumentales. Celles-ci sont surmontées des dais de calcaire engagés dans le mur. Chaque dais est différent des autres; tous reposent sur des culots à tige coudée et sont ornés de petits motifs d'architectures que l'on retrouve également sur les dais de Tréguier.

Un autre édifice majeur nous intéresse; il s'agit du chœur de Quimper ${ }^{33}$ (fig. 19), probablement à peu près contemporain des nefs de Tréguier et de Saint-Pol. Il présente une élévation dont le parti général est le même que celui décrit plus haut. Les supports sont majoritairement alternés, comme à Tréguier ou à Dol; il s'agit, dans les travées droites, de piles à quatre colonnes engagées, dont les noyaux sont soit monocylindriques, soit polygonaux. Ces derniers évoquent quelque peu les piliers complexes de Tréguier, réalisés au cours de la deuxième campagne de travaux ${ }^{34}$. Le triforium est très proche de celui de Tréguier : court, aveugle ${ }^{35}$, souligné par

33. BARRIE, Roger, "La construction de la cathédrale de Quimper ", Mémoires de la Société d'Histoire et d'Archéologie de Bretagne, t. LXIV, 1987, p. 443-453, et INQUELLO, Pascale, La cathédrale Saint-Corentin de Quimper, voies de recherche, DEA d'histoire de l'art, Université Paris IV, 1996.

34. À Tréguier cependant, les noyaux des piliers sont dénués de chapiteaux.

35. Dans certaines travées, la galerie disparaît, et les arcatures sont directement plaquées contre un mur plein. 


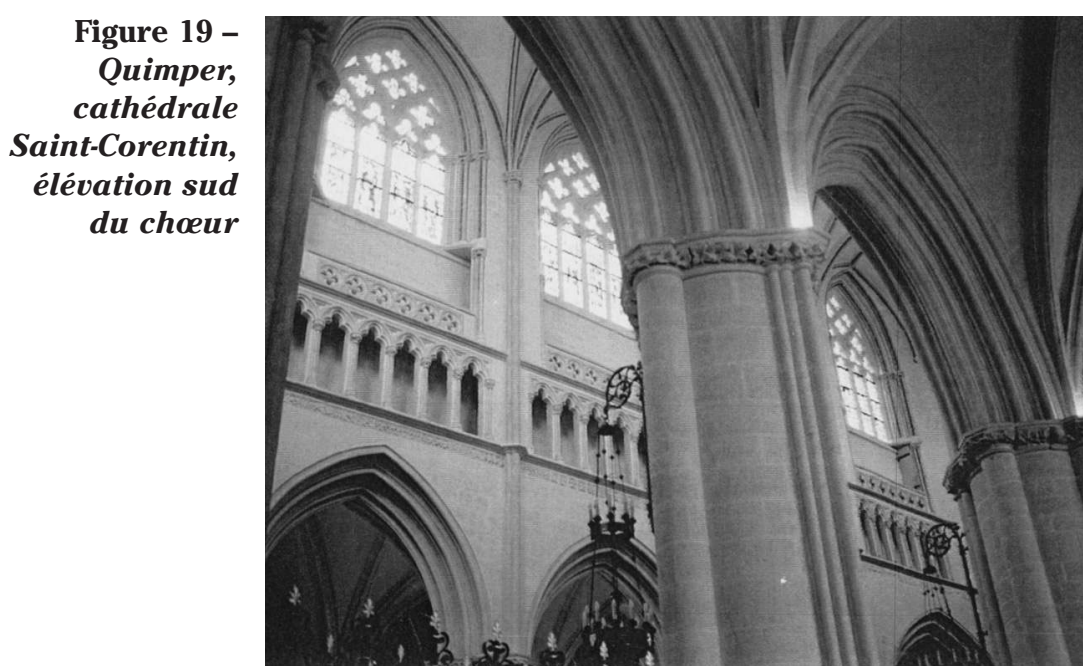

une frise sculptée, il possède des arcatures trilobées prises dans un cadre rectangulaire. Ce n'est donc pas non plus un triforium continu, bien qu'à Quimper la surface murale qui prend place de part et d'autre de ces cadres soit moins importante qu'à Tréguier. Le dernier niveau appartient à la même famille : le mur des fenêtres hautes est épais et pourvu d'une galerie, les fenêtres à remplages sont plutôt courtes et les ébrasements assez prononcés. Quelques détails caractérisent cependant le chœur de Quimper : l'ébrasement est souligné par des colonnettes et des arcs moulurés, et une balustrade longe la galerie, sous les fenêtres; enfin, les arcatures latérales ont été omises, sauf dans la première travée nord-ouest : deux arcatures jumelées prises dans un cadre rectangulaire chanfreiné prennent place à droite de la fenêtre, évoquant ponctuellement les dispositions présentes à Saint-Pol ou à Tréguier.

Construit à partir de $1354^{36}$, le chœur très court de Saint-Brieuc présente une élévation et des proportions proches de celles évoquées jusqu'ici. Au-dessus de piles composées de colonnes séparées par des gorges, les arcs des grandes arcades sont simplement chanfreinés, comme dans les nefs de Tréguier et de Dol; le triforium aux arcatures trilobées est aussi pris, dans chaque travée, dans un cadre rectangulaire. Cependant, une balustrade a été intégrée à ce triforium et occupe la moitié inférieure de sa hauteur, à la manière de certains édifices normands de la deuxième moitié du XIII ${ }^{\mathrm{e}}$ siècle et des siècles suivants ${ }^{37}$. La paroi, épaisse, percée par les fenêtres hautes, est traversée par une galerie, mais ici, comme c'est

36. Couffon, René, " La cathédrale de Saint-Brieuc ", Congrès archéologique de France, $107^{\mathrm{e}}$ session (Saint-Brieuc), 1949, p. 9-33.

37. Un très grand nombre d'églises normandes, marquées par l'architecture rayonnante, présentent cette caractéristique, comme la cathédrale de Sées, l'abbatiale SaintOuen de Rouen, etc. 
aussi le cas pour le chœur de Dol, les fenêtres ne sont pas ébrasées et, comme à Quimper, une balustrade longe la galerie. Enfin, on retrouve la grande variété des dessins des réseaux qui caractérisait la plupart des édifices que nous avons décrits.

\section{Figure 20 - Tréguier, cathédrale Saint-Tugdual, élévation nord-est du chour}

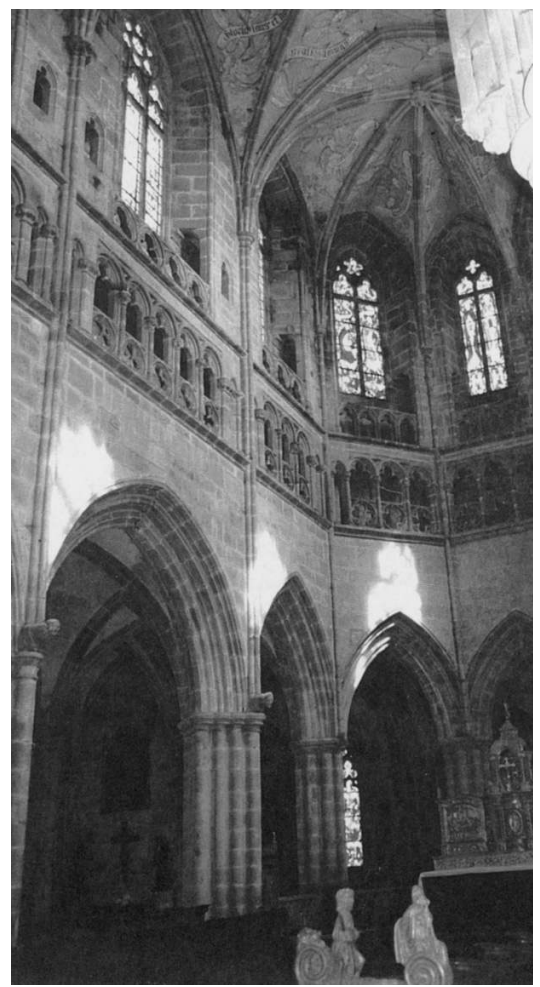

Figure 21 - Quimper, cathédrale Saint-Corentin, élévation nord de la nef

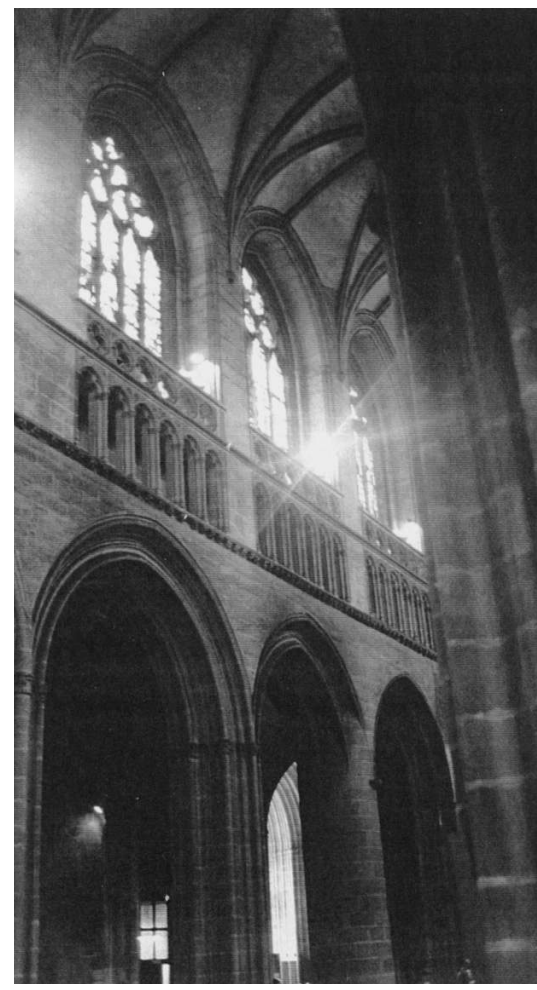

Le chœur de Tréguier, commencé dans le dernier quart du XIV ${ }^{\mathrm{e}}$ siècle $^{38}$ (fig. 20), se rattache à la même tradition architecturale. Certains éléments font directement écho à la nef du même édifice; ainsi, les colonnettes qui scandent l'élévation et reposent sur des marmousets, peuvent être des évocations des culots d'une partie des travées de la nef; le triforium reprend le parti présent à l'ouest, les arcatures trilobées étant prises dans un cadre rectangulaire. Cependant, de part et d'autre de ce cadre, deux arcatures plus petites et indépendantes viennent flanquer la composition centrale, évoquant plutôt le motif du triforium de la nef de Saint-Pol. D'autres influences peuvent être discernées, comme la balustrade réalisée à ce niveau, qui se retrouve à Saint-Brieuc. Au-dessus, les fenêtres sont identiques à celles

38. CORNon, Raymond, « La cathédrale de Tréguier », art. cit., p. 104-105. 
de la nef; seules variantes, la présence de la balustrade qui longe la galerie au devant des fenêtres, et la taille réduite des arcatures latérales, qui ne descendent pas jusqu'à la base de ce niveau. Enfin, les remplages, là encore au dessin très varié, présentent des meneaux transversaux qui ne peuvent manquer d'évoquer les remplages anglais.

Figure 22 - Saint-Pol-de-Léon, cathédrale, mur gouttereau nord du chour

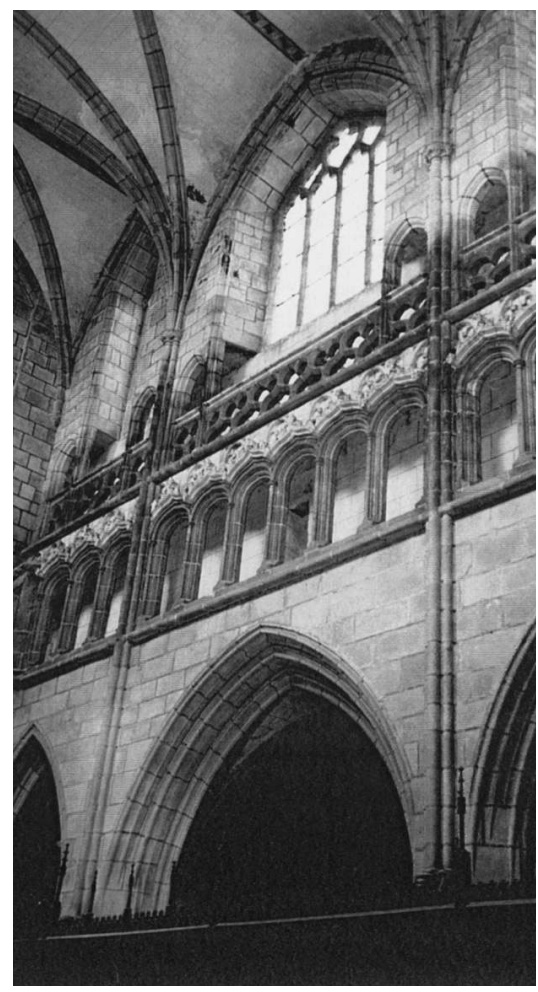

Figure 23 - Lamballe, église Notre-Dame, élévation nord du chour

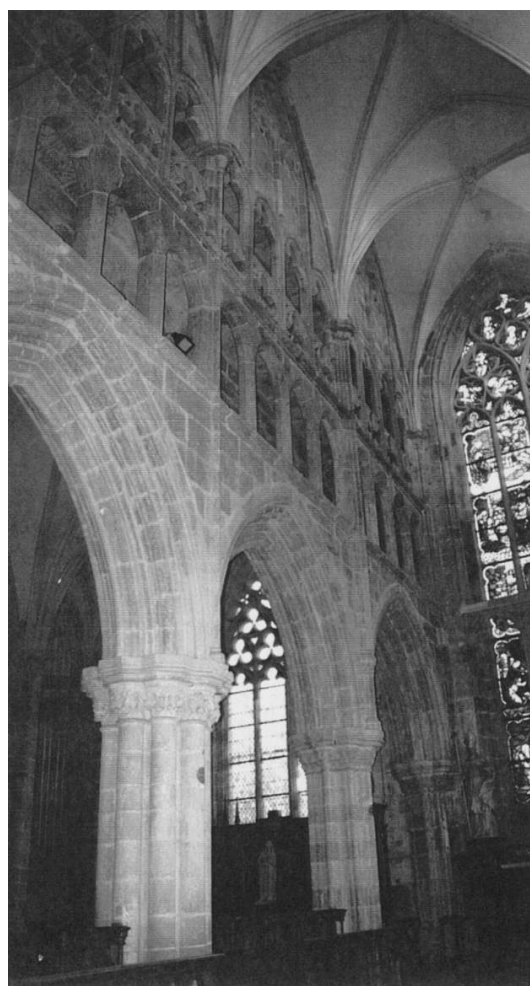

La nef de Quimper (fig. 21), commencée par la façade en $1424^{39}$, est encore représentative de ce courant architectural. Les piliers sont ici encore alternés, bien que différents de ceux du chœur du même édifice et de ceux de Tréguier. Le triforium est presque identique à celui du chœur de même que les fenêtres hautes, mais celles-ci ne sont pas ébrasées. Un détail modifie cependant profondément l'allure de cette élévation : la scansion régulière par les colonnettes de fond à été entièrement omise.

Enfin, à Saint-Pol de Léon (fig. 22), le chœur, commencé à partir de $1431^{40}$, reste fidèle à ce parti. Il est en granite, et non en calcaire comme la

39. InQUello, Pascale, La cathédrale Saint-Corentin de Quimper, art. cit., p. 28.

40. LEGUAY, Jean-Pierre, Un réseau urbain au Moyen Âge..., op. cit., p. 192. 
nef. Le triforium y est toujours court et aveugle, mais ici, les arcatures, toutes identiques, adoptent un rythme continu et sont enrichies par des inclusions de calcaire selon une mode bien répandue dans les pays granitiques. Bien que les fenêtres ne soient pas ébrasées et qu'une balustrade vienne occuper toute la partie inférieure de ce niveau, le parti général est celui de la nef avec, en particulier, la présence des petites arcatures latérales qui flanquent la fenêtre.

Une étude plus exhaustive ne manquerait pas d'évoquer toutes les églises plus modestes de ces régions, dans lesquelles se retrouve tel ou tel trait de ces grandes cathédrales bretonnes. Par exemple, le chœur de NotreDame de Lamballe ${ }^{41}$, réalisé au moins en deux campagnes de construction, et qui présente, au nord (fig. 23), un triforium court et aveugle, aux arcatures simplement chanfreinées; celui-ci est placé sous un deuxième triforium, cette fois aux arcs trilobés et dans lequel prend place une balustrade. Enfin, dans les lunettes de la voûte, un réseau rayonnant évoquant celui des fenêtres hautes est plaqué sur le mur étrangement aveugle. Au sud, le

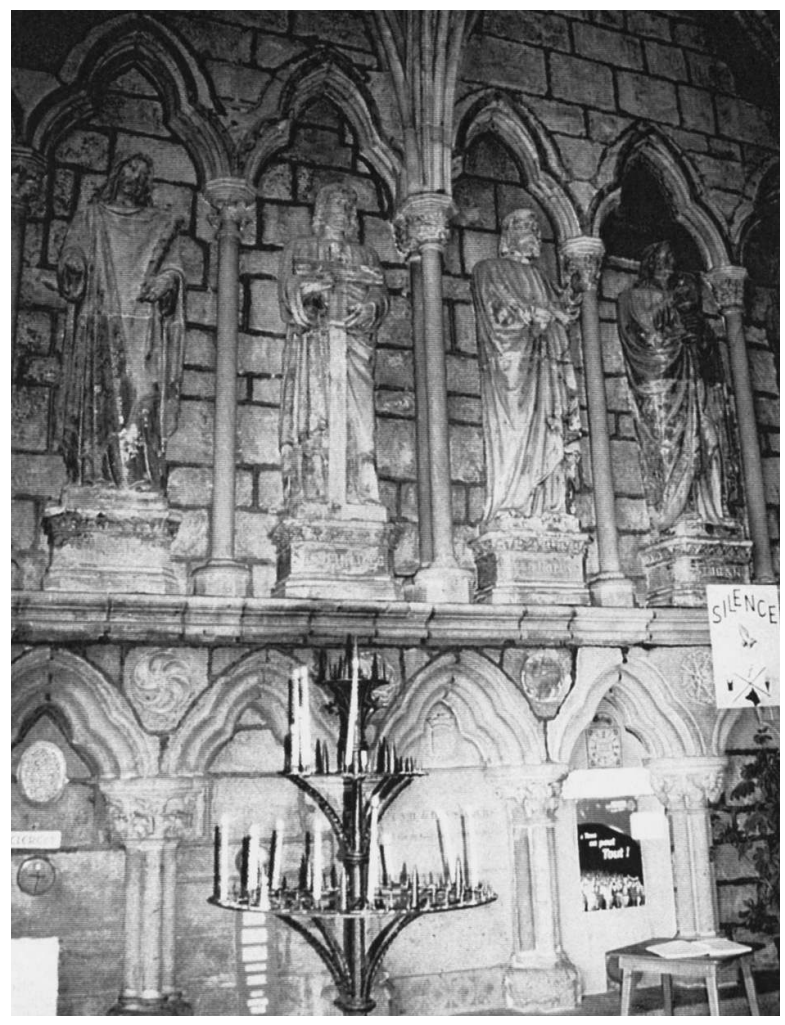

Figure 24 -

Guingamp, église Notre-Dame, porche nord de la nef

41. Mussat, André, « Notre-Dame de Lamballe », Congrès Archéologique de France, 107e session (Saint-Brieuc), 1949, p. 34-55. 
parti est plus classique : seul un triforium à arcatures trilobées et balustrade imbriquée apparaît, surmonté de baies minuscules creusées dans un mur très massif, mais dénué de galerie. Aucun de ces motifs n'est original, tous ayant été empruntés aux grandes églises bretonnes, et seul l'agencement est inédit et le fruit de l'imagination de chaque architecte.

Cette élévation méridionale de Lamballe se retrouve dans la nef de Guingamp ${ }^{42}$, probablement contemporaine, où, au nord, le dernier niveau éclairé est cependant dédoublé par une galerie. L'élévation plus tardive, visible au sud, qui est issue d'une reprise du XvI ${ }^{\mathrm{e}}$ siècle, présente au-dessus des grandes arcades deux galeries superposées, évoquant l'ordonnance originale de l'élévation nord du chœur de Lamballe; le niveau des fenêtres hautes a ici complètement disparu. Nous ne pouvons enfin manquer d'évoquer le grand porche de Guingamp (fig. 24), greffé sur le flanc nord de la nef ${ }^{43}$, et aujourd'hui profondément remanié. Il présente une série de statues d'apôtres en ronde-bosse, placées sous des arcatures trilobées engagées dans les parois, et probablement, à l'origine, un portail proche de ceux que nous avons observés jusqu'ici ${ }^{44}$. Cette œuvre monumentale appartient à la grande famille des porches bretons que nous avons évoqués ici, porches qui constituent l'un des éléments de définition de l'architecture gothique bretonne de la fin du Moyen Âge.

Le parti si original de la nef de Tréguier n'est donc pas unique, mais illustre bien le style gothique régional. En effet, à partir du milieu du XIII ${ }^{\mathrm{e}}$ siècle, et tout au long du siècle suivant, un style gothique propre à la Bretagne s'est défini, dont certaines formes ont persisté jusqu'au début du XVI ${ }^{\mathrm{e}}$ siècle. Cette architecture gothique a été élaborée à partir de quelques motifs continentaux, aussi bien romans que rayonnants; mais elle semble s'être surtout nourrie de l'architecture anglaise de la deuxième moitié du XIII ${ }^{\mathrm{e}}$ siècle et du début du siècle suivant, qui avait intégré quelques formes rayonnantes à des édifices par ailleurs fidèles à une forte tradition romane. Les différentes facettes de cette architecture si particulière, les datations plus précises des édifices, les variantes développées à la fin du Moyen Âge dans diverses régions de Bretagne, et enfin l'interprétation qui en a été faite dans les églises dites secondaires, demandent maintenant à être approfondies, afin que l'importance et l'originalité en soient enfin reconnues.

42. Merlet, François, "Notre-Dame de Guingamp ", Congrès Archéologique de France, $107^{\mathrm{e}}$ session (Saint-Brieuc), 1949, p. 236-256 et MusSAT, André, "Un monument clé : NotreDame de Guingamp ", Bretagne, Architecture et identités, 1997 (rééd. de l'art. paru dans les Mémoires de la société d'histoire et d'archéologie de Bretagne, t. LVI, 1979), p. 354-370.

43. Ibid., p. 358.

44. Ce portail a été profondément transformé, mais il est intéressant de remarquer, au revers de ce portail, des motifs de fleurs (bowl flowers) qui renvoient, là encore, à l'Angleterre. 


\section{RESUME}

La nef de la cathédrale de Tréguier est un bon exemple de l'architecture gothique religieuse qui s'est développée en Bretagne à partir de la deuxième moitié du XIII ${ }^{\mathrm{e}}$ siècle et au cours des siècles suivants. Malgré de nombreux tâtonnement, qui témoignent des recherches alors en cours, le parti général de la nef de Tréguier est singulier en France, et évoque à la fois les effets de masses de l'architecture romane normande et le graphisme des formes rayonnantes. Cette combinaison particulière n'est pas parfaitement originale puisqu'elle caractérise l'architecture gothique anglaise, qui semble constituer une véritable source d'inspiration pour les Bretons. Cependant, parce qu'elles se retrouvent de manière presque systématique dans la plupart des édifices gothiques de Bretagne, les différentes formules retenues à Tréguier déterminent véritablement un style régional.

\section{ABSTRACT}

The nave of Tréguier cathedral is a good example of the ecclesiastical gothic architecture that developed in Britanny between the first half of the XIIIth century and the end of the medieval period. In spite of hesitations, which show that experiments were made, the architecture of the nave of Tréguier was new to France. It evokes both the massive architecture of the Norman Romanesque and the refinement of the Rayonnant. This combination was not really original for it occurred in England at the same time, and it appears that the English gothic strongly influenced the architects of Tréguier. However, since the particular vocabulary of the nave of Tréguier can be seen in the cathedrals and smaller churches of this region, we can say that it determined a very specific gothic style in Brittany. 